\title{
DCCP-based Multiple Retransmission Technique for Multimedia Streaming
}

\author{
Árpád Huszák \\ Budapest University of Technology and Economics \\ Magyar Tudósok krt.2. \\ $\mathrm{H}-1117$ Budapest, Hungary \\ +36 1 463-3227 \\ huszak@hit.bme.hu
}

\author{
Sándor Imre \\ Budapest University of Technology and Economics \\ Magyar Tudósok krt.2. \\ $\mathrm{H}-1117$ Budapest, Hungary \\ +36 1 463-3256 \\ imre@hit.bme.hu
}

\begin{abstract}
Retransmission-based error recovery is the simplest technique to minimize the overall packet loss ratio in order to increase the quality of the applications. Multimedia applications are becoming increasingly popular in IP networks, while in mobile environment the limited bandwidth and the higher error rate arise in spite of its popularity. Retransmission can be also used for loss recovery in media applications, but the number of retransmissions is limited by the playout buffer and the recent network delay.

In this paper we present a source controlled adaptive multiple retransmission scheme for multimedia streaming. Due to source controlling the receiver do not need to send additional retransmission request messages. The other advantage of transmitter controlled decision algorithm is that the necessary RTT information is available at the source due to DCCP transport protocol. Therefore no additional network measurements and probe messages are needed. With the knowledge of the actual network delay and the playout buffer delay, we are able to determine the number of retransmission attempts without late delivery of packets.
\end{abstract}

\section{Keywords}

Multimedia, streaming, retransmission, DCCP

\section{INTRODUCTION}

The delay sensitive real-time multimedia applications are gaining prominence in IP-based wired and wireless networks. Retransmission-based error recovery is often considered inappropriate for multimedia applications, because of its latency. However, this solution can be attractive because it requires minimal network bandwidth and processing cost as well as improvement of the video quality. Despite its latency,

Permission to make digital or hard copies of all or part of this work for personal or classroom use is granted without fee provided that copies are not made or distributed for profit or commercial advantage and that copies bear this notice and the full citation on the first page. To copy otherwise, or republish, to post on servers or to redistribute to lists, requires prior specific permission and/or a fee.

MoMM 2008, November 24-26, 2008, Linz, Austria

(c) 2008 ACM 978-1-60558-269-6/08/0011 $\$ 5.00$ retransmission can be used successfully in many cases, especially if playout buffering is employed. For the retransmission to be successful, retransmitted packet must arrive at the receiver in time for playback. To minimize the probability of wastefully retransmitted packets, a playout buffer is usually set up at the receiver side to prefetch a certain amount of data before playback. The buffered data provides additional time to absorb the retransmission delay making the retransmission acceptable for one-way pre-recorded and oneway live media applications.

Data transmission on high error rate channels can cause multiple loss of the same packet, when the lost packet is retransmitted again and again. In wireless mobile networks packet loss can occur not only due to wireless errors, but due to congestion as well. In a congested network the round-trip-time (RTT) and the loss probability is significantly higher and the retransmitted packets probably will be lost again, therefore the retransmission of lost video stream packets is not recommended in such conditions. The key issue is how to set up a limitation for the retransmissions to avoid late reception of the packet and minimize the wastefully transmitted packets.

In our scheme the decision of retransmissions is based on the network delay. To make the decision effective recent information are needed about the network, because theses network properties (delay, available bandwidth, loss rate, etc.) can vary intensively [1]. In some situations the packet can be retransmitted more times after it is lost, but the number of possible retransmissions is changing due to varying network conditions. In order to get the latest information and make the best decision we rely on the transport layer.

Loss-tolerant real-time multimedia applications prefer UDP or UDPLite [2] but in our proposal we applied the unreliable DCCP (Datagram Congestion Control Protocol) [3] as transport protocol because it uses sequence numbering, acknowledgements and congestion control algorithms. Sequence numbers and acknowledgement are needed to identify the lost packets while the congestion control algorithms (TCPLike Rate Control [4] and TFRC (TCP Friendly Rate Control) [5]) manage the actual round-trip-time (RTT) measurements. The actual RTT plays a very important role to decide weather to retransmit a packet. In our proposal the transmitter determines whether to retransmit a packet, based on the DCCP inner RTT 
parameter. With this technique no additional network measurements and probe messages are needed.

The rest of the paper is organized as follows. In Section II we present background and related work. . In Section III we introduce our source controlled selective retransmission method for multimedia applications. The obtained performance results are presented in Section IV. Finally, we summarize our paper and make the conclusions in the last section.

\section{RELATED WORK}

Several proposals have described retransmission schemes for recovering lost streaming media packets. Attempts were made to implement a selective retransmission protocol with a decision algorithm [6]. The decision is made by the Euclidean distance calculated by the loss and latency ratio. This protocol does not use playout buffer and does not implement congestion avoidance mechanisms.

In retransmission-based schemes, upon detection of a gap, the receiver decides whether to send a negative acknowledgement (NACK) based on the current estimate of RTT and the playout time of the missing frame [7]. Sinha and Papadopoulos [8] proposed two retransmission-based error recovery protocols, one for unicast and another for multicast, which do not rely on timers to perform multiple retransmissions. In the scheme introduced in [9], the server decides whether it retransmits packets or not based not only on importance of each packet but also playout time of each packet. The server estimates time to play the packet and time which the packet arrives at the client if it is retransmitted. If estimated time to play the packet is later than the time when the packet arrives at the client, the server does not retransmit the packet. In these proposals the client sends the retransmission request to the server whenever it detects a packet loss.

Some other proposals have investigated selective retransmission methods based on SR-RTP (Real-Time Transport Protocol) [10]. The schemes based on RTP [11][12][13] do not suit the streaming service. If a streaming packet does not arrive at the client until after its playout time, it is discarded by the client. This problem arises because the playout times of the packets are not taken into account. Therefore, the schemes can wastefully deliver late packets. Feamster and Balakrishnan [14] analyzed this approach with SR-RTP. This RTP extension provides semantics for requesting the retransmission of independently processible portions of the bitstream and a means for reassembling fragmented portions of independently processible units. They have shown that, by recovery of only the most important data in the bitstream, significant performance gains can be achieved without much additional penalty in terms of latency.

In most of the related works the receiver controls the retransmission procedure and do not deal with multiple losses and multiple retransmissions of the packets. Bad wireless channel conditions and heavily loaded links can lead to high packet loss ratios. In these situations the retransmitted packets can be also lost and retransmitted again. The corresponding works do not deal with this possibility. In most cases the decision algorithm is implemented at the receiver therefore additional administration messages must be sent to the sender.
Common solution is to use NACKs (Negative ACKnowledgements) or Retransmission Request messages. In our proposal no administration messages are needed because the decision procedure is located at the transmitter. The other advantage of the transmitter side decision is that the input parameters of the decision algorithm (RTT, estimated link bandwidth, etc.) are available at the source using the DCCP transport protocol. The algorithm we introduce in this paper uses DCCP acknowledgements in order to avoid the need for manually generated acknowledgements that are used in other solutions.

\section{THE MULTIPLE RETRANSMISSION TECHNIQUE}

In this section a DCCP-based multiple retransmission scheme is introduced, investigating the effects of multiple losses and retransmissions caused by bad link conditions. The Datagram Congestion Control Protocol (DCCP) is a newly defined transport protocol by the IETF that implements bidirectional, unicast connections of congestion controlled, unreliable datagrams. For our purpose this protocol is ideal because it provides all the needed information for the decision algorithm. The DCCP header contains sequence number field that is indispensable to recognize and identify the lost packets.

Due to high wireless loss and congestion probability the multiple retransmissions can significantly increase the overall delay of the successful packet delivery. This delay limits the number of retransmissions when multiple retransmissions are used. If we want to provide reliable transmission we have to allow multiple retransmissions. In other words, the number of retransmission is infinite. In this case the expected value of the delay $(d)$ due to retransmission can be calculated using the formulas of geometrical progression:

$$
\begin{gathered}
\mathbb{E}(d)=\frac{R T T}{2}(1-p)+\frac{3 R T T}{2}(1-p) p+\frac{5 R T T}{2}(1-p) p^{2}+\ldots= \\
=\frac{R T T}{2}(1-p)\left(1+3 p+5 p^{2}+7 p^{3}+\ldots\right) \\
\mathbb{E}(d)=\frac{R T T}{2}(1-p) \cdot \sum_{n=0}^{\infty}(2 n+1) p^{n}=\frac{R T T}{2} \cdot \frac{1+p}{1-p}
\end{gathered}
$$

If we also consider the loss detection delay $(\tau)$ the expected value of the retransmission delay is given in the following equations.

$$
\begin{aligned}
\mathbb{E}(d)= & \frac{R T T}{2}(1-p)+\left(\frac{3 R T T}{2}+\tau\right)(1-p) p+ \\
& +\left(\frac{5 R T T}{2}+2 \tau\right)(1-p) p^{2}+\ldots \\
\mathbb{E}(d)= & \frac{R T T}{2}(1-p) \cdot \sum_{n=0}^{\infty}(2 n+1+n \tau) p^{n}= \\
= & \frac{R T T}{2} \cdot \frac{1+p}{1-p}+\tau \frac{p}{1-p}
\end{aligned}
$$


According to equations (1) - (4) practically the number of retransmission will be always less then 4 , that is also show in the simulation evaluation in Section IV. Even in case of extremely high overall loss rate (wireless loss + congestion loss) of $20 \%$, the probability that the packet was lost and retransmitted four times is about $0.1 \%$. The Figure 1 depicts the distribution of the number of retransmissions for different losses.

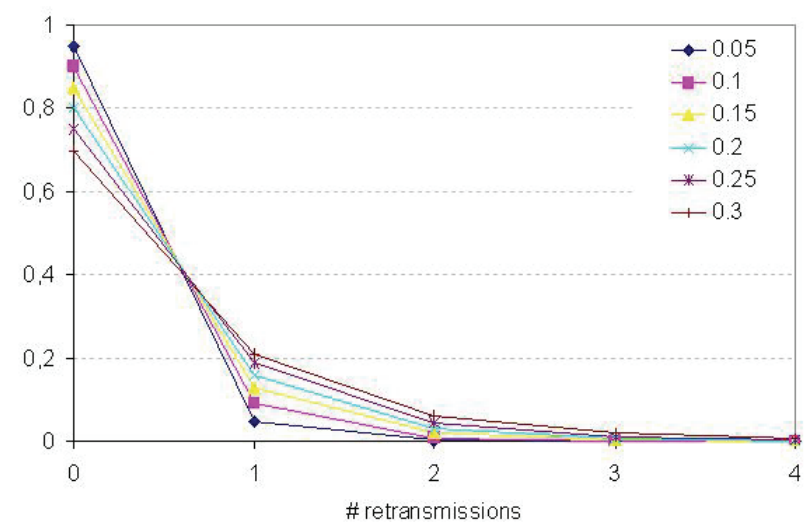

Figure 1. Distribution of the number of the loss and retransmission of the same packets

The number of retransmission is limited by the playout buffer delay installed at the receiver and the network delay. In order to control the retransmission process at the source side, the transmitter must know the actual network and playout buffer delay. The DCCP transport protocol manages the actual roundtrip-time (RTT) measurements to ensure the proper operating of the TFRC congestion control algorithm. Our multiple retransmission scheme shall retrieve the value of actual RTT from the DCCP socket using the getsockopt() system call. The playout buffer delay can be also managed using the Flood method introduced in [15].

The Flood method heaps up some data after the connection setup and transmits it all together after desired buffer delay time. With this method the receiver will receive more data then it should process therefore certain amount of data will be heaped up in the receiver's playout buffer. The process rate of the receiver is known by the transmitter because it is equal with the bitrate of the current video so the duration of the Flood method can be calculated. The arising question is that how to determine the playout buffer delay to introduce adequate delay for multiple retransmissions. The playout buffer delay should be long enough to make the packet loss detection and the retransmissions feasible but it should be as short as possible.

The purpose of the presented multiple and selective retransmission is to efficiently discard from the retransmission queue those packets that can not be delivered before the playout. The media packets that are arriving late will only waste the network bandwidth. To determinate how time a packet should be lost and retransmitted the following correlations should be considered, where $\mathbb{E}(d)$ is expected value of the transmission delay including the delay due to retransmissions, $p$ is the overall packet loss, $T_{b d}$ is the receiver's playout buffer delay and $\tau$ is the loss detection delay.
Number of possible retransmission is 1 if:

$$
\mathbb{E}\left(d_{1}\right)=\frac{R T T}{2}(1-p)(1+(3+\tau) p)<T_{b d},
$$

and $\mathbb{E}\left(d_{2}\right)>T_{b d}$.

Number of possible retransmission is 2 if:

$$
\mathbb{E}\left(d_{2}\right)=\frac{R T T}{2}(1-p)\left(1+(3+\tau) p+(5+2 \tau) p^{2}\right)<T_{b d},
$$

and $\mathbb{E}\left(d_{3}\right)>T_{b d}$.

Number of possible retransmission is $n$ if:

$$
\mathbb{E}\left(d_{n}\right)=\frac{R T T}{2}(1-p) \sum_{i=0}^{n}(2 i+1+i \tau) p^{i}<T_{b d}
$$

and $\mathbb{E}\left(d_{n+1}\right)>T_{b d}$

The upper limit of the loss detection delay $(\tau)$ is determined by the TFRC congestion control algorithm used in DCCP because this algorithm specifies the frequency of the DCCP acknowledgements. Figure 2. depicts DCCP transmission sequence and a retransmission with gap detection to discover the lost packets. In the figure $f_{p}$ is the frequency of packet sending so the elapsed time between two packets is $1 / f_{p}$.

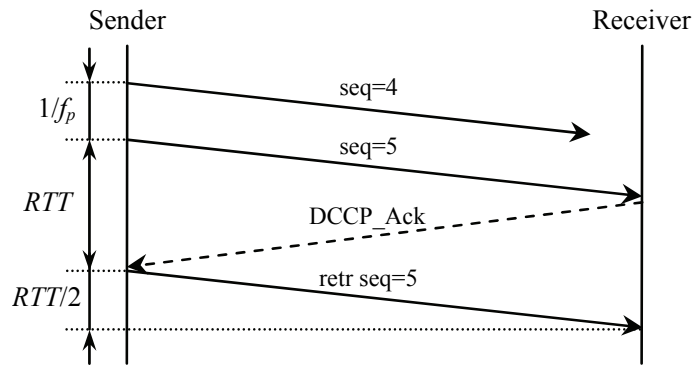

Figure 2. DCCP transmission sequence

The receiver sends DCCP-Ack packets at least once per RoundTrip-Time acknowledging the data packets, unless the sender is sending at a rate of less than one packet per RTT, as indicated by the TFRC specification [RFC 3448]. According to the specification the loss detection delay is:

$$
\frac{1}{f_{p}}<\tau<R T T
$$

The equations (5) - (7) give theoretical correlations about the multiple retransmission possibilities. In practice the decision whether to retransmit a once or a more time lost packet is made real time at every loss occurrence. The decision algorithm must know the remained playout buffer time for the each media packet. The actual value of the loss detection delay can vary therefore the first sending time of the packet $\left(t_{1}\right)$ and the acknowledgement reception time $\left(t_{0}\right)$ is used to determine the elapsed time $\left(T_{E}\right)$. The acknowledgement packet informs the transmitter whether the sent packet arrived successfully or not. 


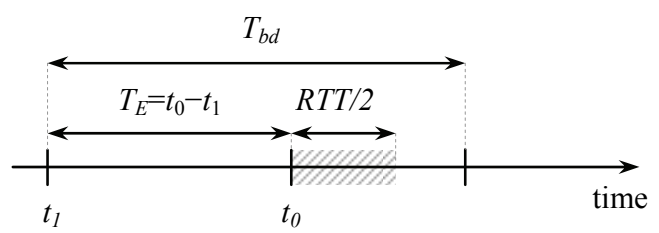

Figure 3. Time sequence. Packet transmission, and the acknowledgement reception (loss detection) times determinates the remained time for the retransmission.

During the $T_{E}$ elapsed time period the sent packet can be lost and retransmitted more times, therefore the loss detection times are already included in $T_{E}$. For each packet one $t_{1}$ time is assigned, while more acknowledgement reception can occur in case of multiple losses. After receiving an acknowledgement at $t_{0}$, that contains the sequence numbers of lost packets, the transmitter should decide which packet is worth to retransmit. The decision algorithm calculates the remaining time that must be more then the actual one-way network delay (RTT/2) for successful retransmission, which is available at the transmitter due to DCCP's RTT measurements. A lost packet should be retransmitted if

$$
T_{b d}-\left(t_{0}-t_{1}\right)>\frac{R T T}{2} .
$$

In some cases the RTT can be so high that even the first transmission can not be successfully accomplished. Therefore the decision is made not only when a packet is lost, but at every packet transmission. When sending a new media packet $t_{1}$ and $t_{0}$ is considered to be equal, that means a packet can be sent when

$$
T_{b d}>\frac{R T T}{2} .
$$

The effectiveness of the multiple retransmission method highly depends on the playout buffer size and its delay. The optimal playout buffer setup is out of the scope of this paper, however numerous investigations were made on this issue [15]-[18]. The upper bound of $T_{b d}$ is the maximal acceptable delay that is few seconds in the case of one-way live media applications but it should not be higher then tolerance of the user for two-way interactive applications. The perceptual tolerance of the user is around $150-200 \mathrm{~ms}$ [19]. We note that the playout delay can be much larger in case of one-way stored media retrieval (e.g., video-on-demand) without any adverse effect on the perceived quality. In practice the playout delay may be limited by the desired delay for control operations like fast-forward, pause and also for multiple retransmissions. In general the playot buffer delay setup is approximately $0.5-1$ second. In the retransmission point of view the lower bound of the playout buffer delay is determined by the network delay.

Retransmitting packets will also increase the bandwidth used for the video stream transmission. The additional load highly depends on the overall packet loss ratio. The expected value of the overall bandwidth can be calculated as follows, where $\mu$ is the video bitrate, $\mu^{\prime}$ is the additional bitrate due to retransmissions and $p$ is the loss probability:

$$
\mathbb{E}\left(\mu+\mu^{\prime}\right)=\mu+\mu p+\mu p^{2}+\ldots=\mu+\mu \frac{p}{1-p}=\mu \frac{1}{1-p}
$$

\section{RESULTS AND ANALYSIS}

The performance of the multiple retransmission technique has been explored in Ns2 [20]. In the simulations a dumbbell topology with $1 \mathrm{Mbps}$ links and $10 \mathrm{~ms}$ link delays were used. The bottleneck link was considered to be the wireless link, in order to introduce wireless packet losses using a simple random drop model with the given loss probability. The loss probability on link S-R was set between $0.1 \%$ and $10 \%$.

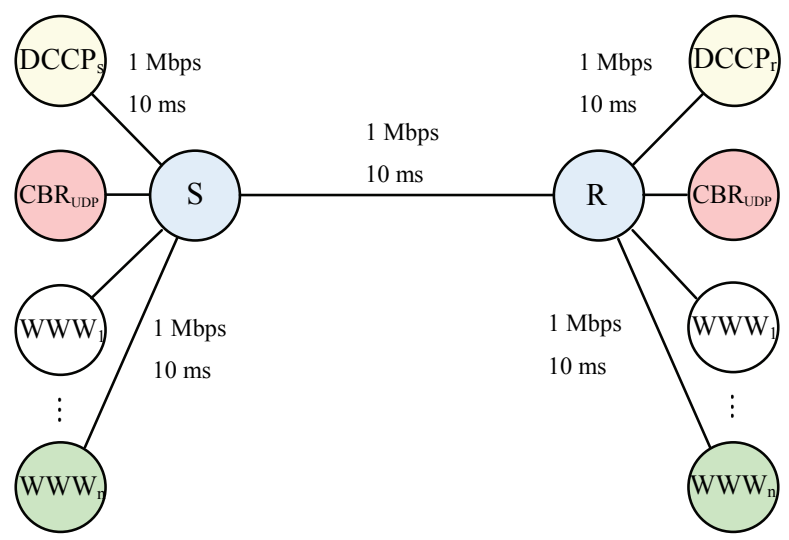

Figure 4. The dumbbell network topology

In the simulations WWW and CBR (Constant Bit Rate flow) over UDP background traffic were set up. In order to simulate the effects of a heavy loaded network the number of WWW users was between 5 and 40. The WWW traffic was generated based on Pareto distribution with average page sizes of $30 \mathrm{kB}$ (shape 1.5), while the frequency of new WWW page requests were following the Exponential distribution with 5 seconds average waiting times. The duration of the simulations was $200 \mathrm{~s}$, while from $0.1 \mathrm{~s}$ to $100 \mathrm{~s}$ a $300 \mathrm{kbps}$ CBR/UDP connection was set up. The purpose of the background traffic was to continuously change the conditions of the bottleneck link causing high variations in the measured RTT.

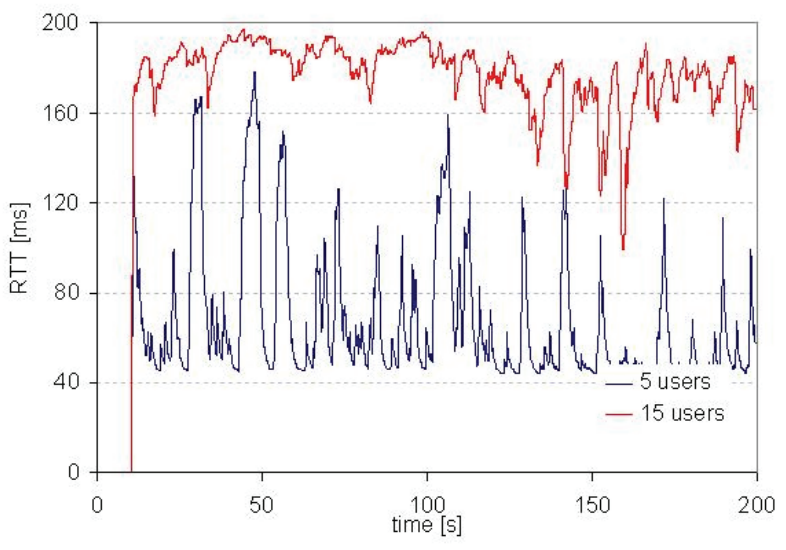

Figure 5. RTT variation measured by the DCCP-based multiple and selective retransmission 
In the simulations we have studied how the DCCP based multiple retransmission method can perform between such conditions. The effectiveness of the proposed technique can be appreciated according the level of the user's satisfaction. To analyze the quality of the 300kbps MPEG-4 video stream, the PSNR (Peak Signal to Noise Ratio) objective quality parameter was used. We used a constant bitrate video stream in the simulations but of course our method is applicable for variable bitrate streams too.

In the first testcases the packet loss evaluation was done. The $1 \mathrm{MB}$ bandwidth on the bottleneck link is maximally utilized, therefore frequent packet losses happen due to congestion, while the additional packet losses due to channel errors increase further the number of lost packets. The retransmissions can improve the overall packet ratio, where the playout buffer size plays important role. As high the playout buffer delay is as more lost packet can be retransmitted again and again. In Figure 6 the ratio of the number of lost packets and the total number of transmitted packets is shown. Of course the retransmissions significantly reduce this ratio.
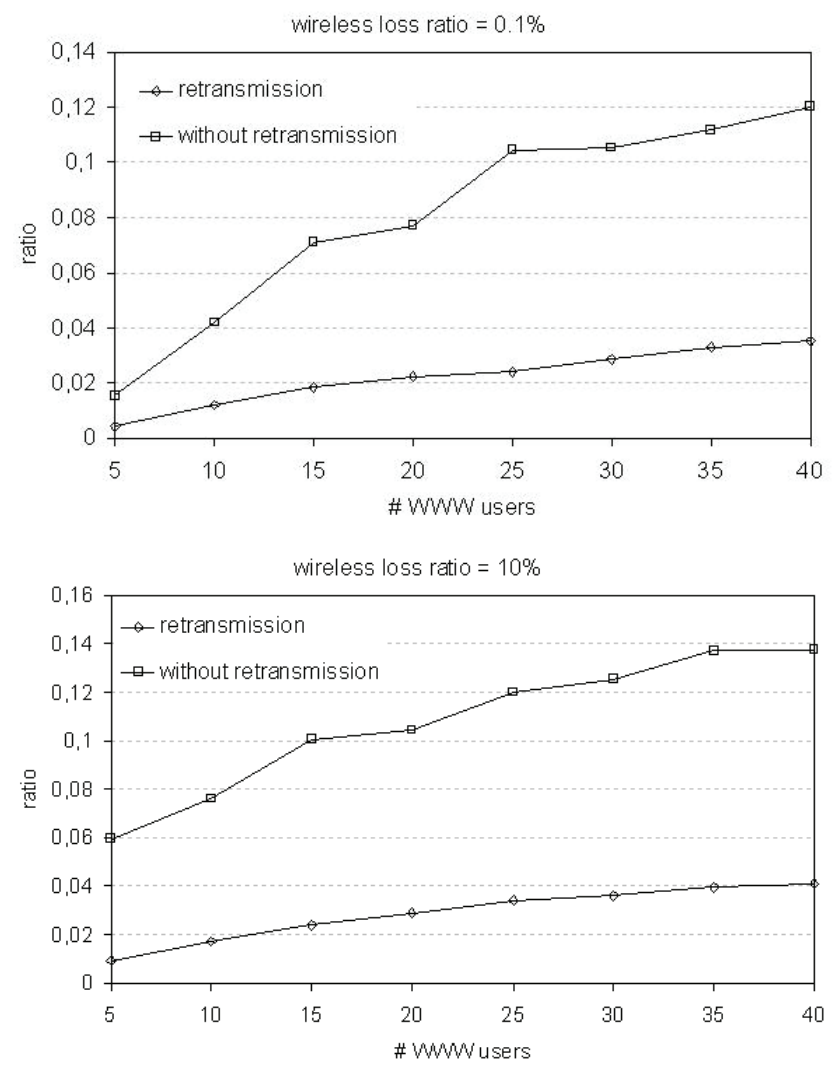

Figure 6. The average packet loss ratios in function of the number of WWW users (level of congestion). The bottleneck link loss probabilities are set to $0.1 \%$ and $10 \%$.

In the simulations a playout buffer was set up which was between $100 \mathrm{~ms}$ and $1500 \mathrm{~ms}$. Each point in Figure 6 is calculated as the average of the measured loss ratios in case of different playout buffer delay setups. In Figure 7 also the loss ratio is pictured but in this case the selective retransmission technique was used.

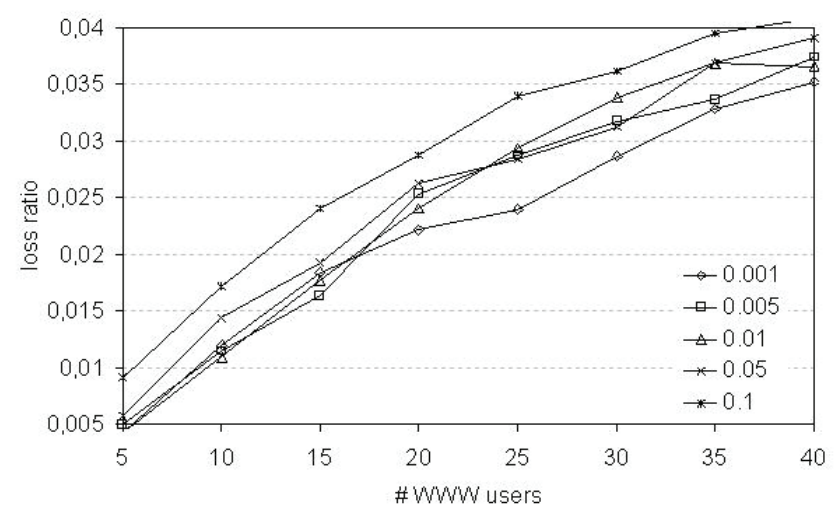

Figure 7. The average packet loss ratios using retransmissions

The narrow bandwidth bottleneck link and the high link utilization lead to significant packet losses. The measured RTT and congestion level are correlated. In case when the network is congested and frequent packet losses occur, the RTT become very high, because the buffers of the network routers become full. When the RTT is high the retransmission will be probably disabled, because there will be not enough time for the retransmitted packet to arrive in time. It is desirable to disable the retransmission when the network is congested, because these packets should be lost again or cause the loss of other packets. In order to analyze the effect of different playout delay setups the loss ratio was calculated. The result is introduced in Figure 8.

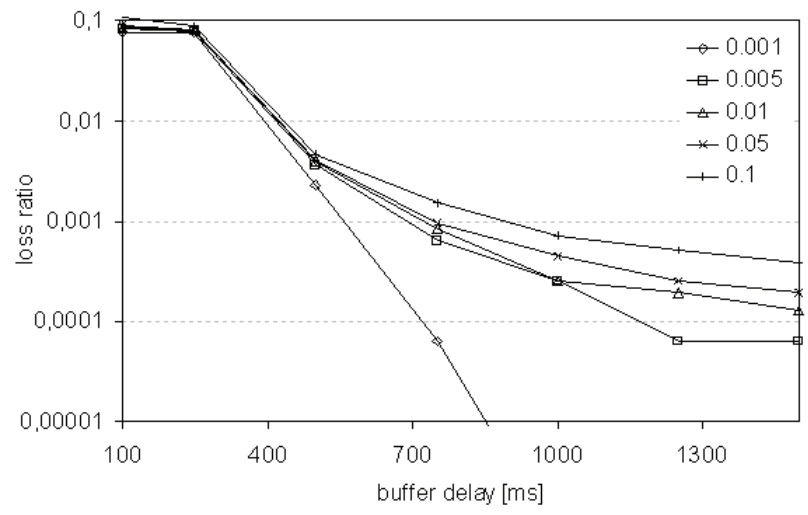

Figure 8. The average packet loss ratios in function of buffer delay setup

Increasing the delay of the playout buffer more retransmissions can be managed. As Figure 8 shows when the playout delay is low the retransmission can not be successfully accomplished. In case of $100 \mathrm{~ms}-500 \mathrm{~ms}$ playout buffer delay setups only few retransmissions can be done, because the RTT can be higher then $200 \mathrm{~ms}$ when the number of WWW users is high. The figure shows that even at $0.1 \%$ link loss probability (due to channel errors) the packet loss ratio rises up to $7 \%$. The reason is that some packets were transmitted not once. Before transmitting a packet the RTT is examined and the packet is discarded from the sending queue when the RTT is too high. 
The playout delay and the actual Round-Trip-Time will determinate how many times can be a packet retransmitted after multiple losses and retransmissions. The simulations confirm the theoretical calculations when examining the maximum number of retransmissions. The possibility of loosing and retransmitting a packet four times is negligible. Even three time retransmission possibility is very low. The next figure depicts the distribution of retransmission attempts considering the playout buffer delay limitations.

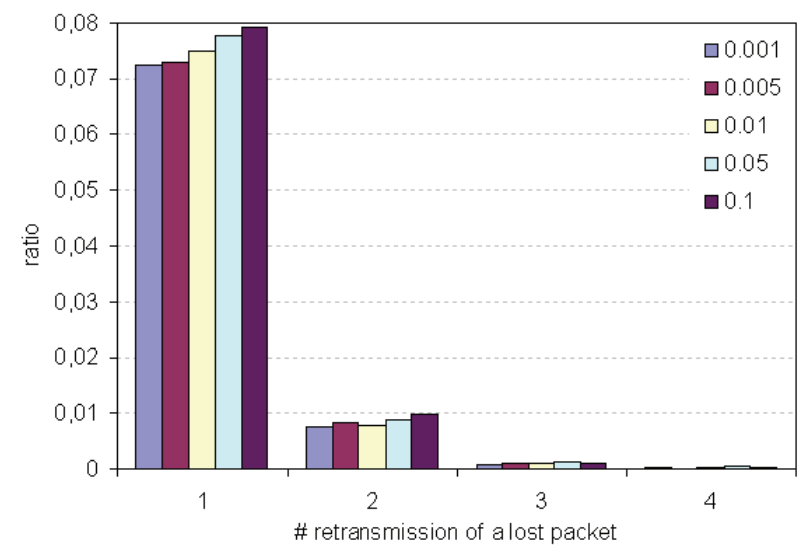

Figure 9. Distribution of retransmission attempts

The empirical probability of retransmitting a packet three times is about $0.1 \%$, while having four retransmissions is $0.01 \%$. These values are calculated as the average of 7 measurements where the playout buffer was set between $100 \mathrm{~ms}$ and $1500 \mathrm{~ms}$. The ratio of successful retransmissions was also studied in function of buffer delay.

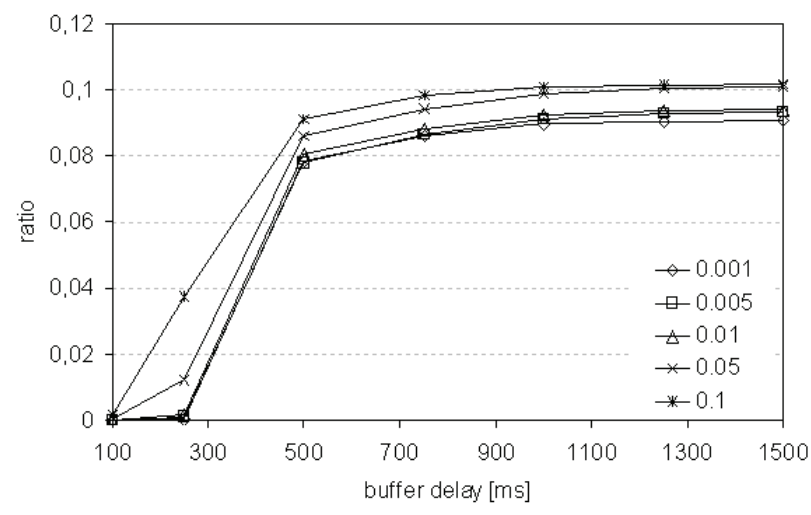

Figure 10. Average ratio of successfully retransmitted packets in function of playout buffer delay

In cases when the RTT significantly increases (e.g. due to congestion) some of the lost packets can not be retransmitted, because the multiple retransmission algorithm will disable it. In our simulations the possibility of enabling the retransmission was higher then disabling it. This is explainable with high number of successful packet delivery for the first sending attempt (see Figure 9). When the load of the bottleneck link is increasing the RTT is raising and fewer packets can be retransmitted when it is lost. The next figure shows the decision ratios of enabling and disabling the retransmission.

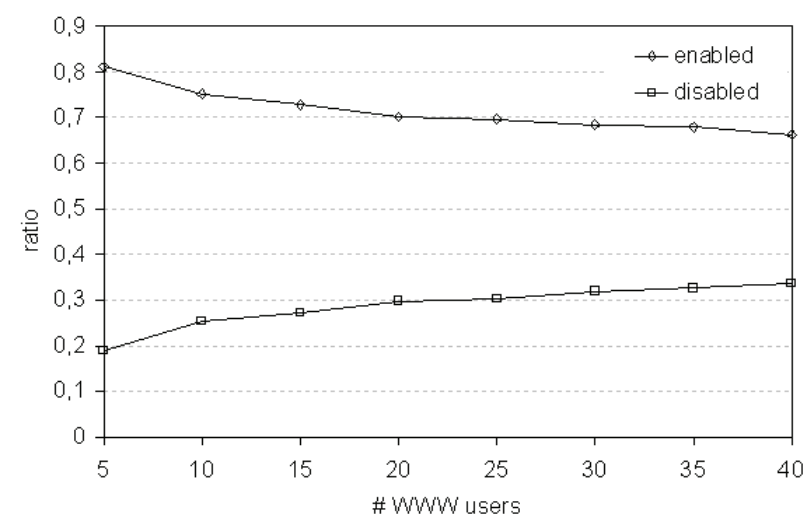

Figure 11. Average ratio of disabling and enabling the retransmissions to all video packets

The presented results up to this point were statistical evaluation of the selective and multiple retransmission scheme. The main goal of our proposal is to improve the quality of video streams. To see the effectiveness our source controlled selective retransmission scheme we measure the average peak signal-tonoise ratio (PSNR) of the transmitted video stream. Peak Signal to Noise Ratio is a coarse and controversial indicator of picture quality that is derived from the root mean squared error (RMSE). The PSNR for a degraded $N_{1} \times N_{2}$ 8-bit image $f$ ' from the original image $f$ is computed by the formula

$$
20 \log _{10} \frac{255}{\left(\frac{1}{N_{1} N_{2}} \sum_{x=0}^{N_{1}-1} \sum_{y=0}^{N_{2}-1}\left[f(x, y)-f^{\prime}(x, y)\right]^{2}\right)^{1 / 2}}
$$

The real improvement can be evaluated analyzing the transmitted MPEG-4 video quality. PSNR values measured against the original frames with varying degrees of packet loss. As the packet loss increases the frame-by-frame PSNR drops dramatically.

The background traffic level significantly reduces the retransmission attempts and increases the overall packet loss. The effect of the network overload on video quality is obvious. The measured average PSNR values are presented in Figure12 in case of multiple retransmission method a) and without it b). As it was more time noted and presented, the number of lost packets as well the video quality highly depends on the playout buffer delay (see Figure 13). The PSNR comparison of the retransmission technique and media streaming without packet retransmissions over heavy loaded links is presented in Figure 14. 


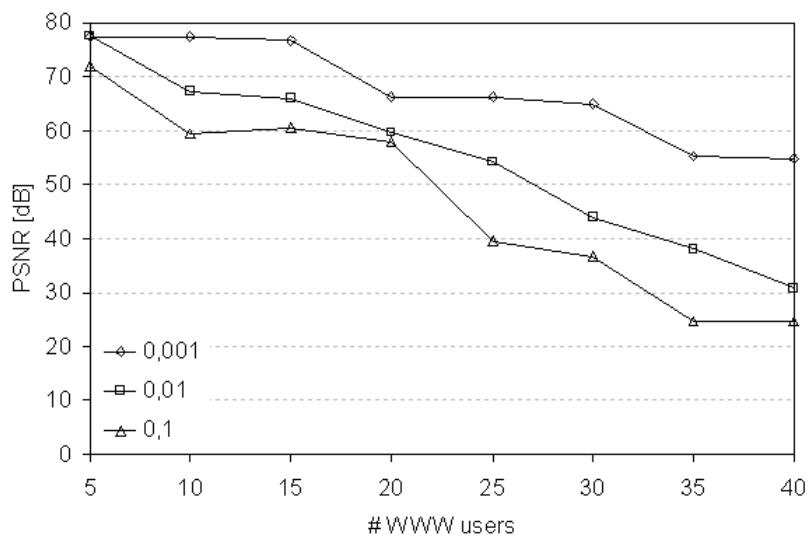

a) with retransmissions

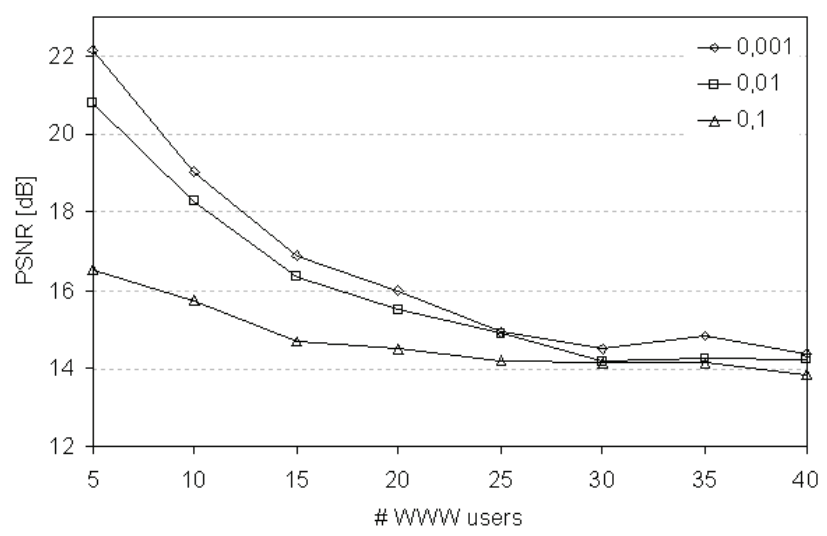

b) without retransmissions

Figure 12. Average PSNR values in function of background traffic

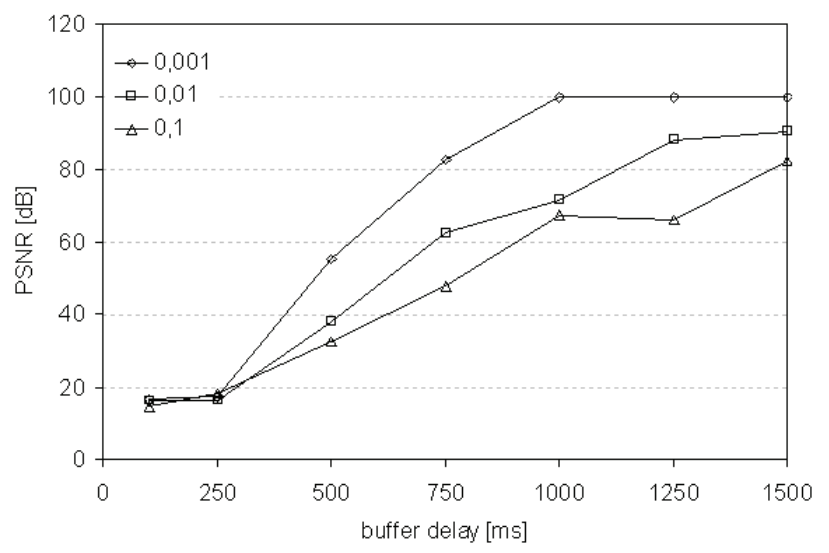

Figure 13. Average PSNR values in function of playout buffer delay

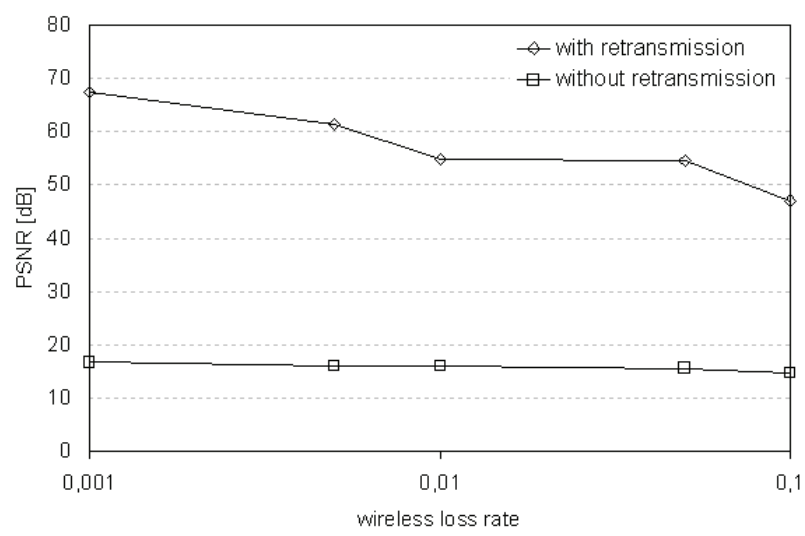

Figure 14. PSNR comparison of retransmission based and UDP-like best effort video streaming technique

To obtain these results no additional messages were needed, because the DCCP transport protocol has provided all the necessary information for the Flood method and for the decision algorithm. Even more the DCCP employs congestion control mechanisms so this is another advantage of our solution.

\section{CONCLUSION}

In this paper, we propose playout time oriented retransmission scheme for multimedia streaming. In our scheme, the server decides whether it retransmits lost packets or not, based on the playout time of each packet. We have analyzed the effects of packet loss on the quality of MPEG-4 video and proposed a model to avoid these effects. From the results of our experiments in which we compare our scheme to the conventional scheme, we show advances of our scheme. Our scheme drastically reduces the number of wastefully retransmitted packets, while ratio of successful retransmitted packets significantly increases the video stream quality. In the future, we will study essential solution to poor ratio of successful retransmitted packets to all retransmitted packets in short delay environment.

\section{ACKNOWLEDGMENTS}

This work was supported by the Mobile Innovation Centre (MIK) and the OPTIMIX project which is partly funded by the Seventh Framework Programme of the European Commission's Information Society Technology.

\section{REFERENCES}

[1] Y. Zhang, N. Duffield, "On the Constancy of Internet Path Properties", Proc. 1st ACM SIGCOMM Workshop on Internet Measurement, November 2001.

[2] L-A. Larzon, M. Degermark, S. Pink, L-E. Jonsson, G. Fairhurst, "The Lightweight User Datagram Protocol", Internet Engineering Task Force, RFC 3828, July 2004.

[3] E. Kohler, M. Handley, S. Floyd, "Datagram Congestion Control Protocol", Internet Engineering Task Force, RFC 4340, March 2006. 
[4] S. Floyd and E. Kohler, "Profile for DCCP Congestion Control ID 2: TCP-like Congestion Control", draft-ietfdecp-ccid2-10.txt, IETF, March 2005.

[5] S. Floyd, E. Kohler, and J. Padhye, "Profile for DCCP Congestion Control ID 3: TFRC Congestion Control", draft-ietf-dccp-ccid3-11.txt, IETF, March 2005.

[6] Mike Piecuch, Ken French, George Oprica, Mark Claypool, "A Selective Retransmission Protocol for Multimedia on the Internet", Proceedings of SPIE International Symposium on Multimedia Systems and Applications, Boston, MA, USA November 2000.

[7] C. Papadopoulos, G. M. Parulkar, "Retransmission-based Error Control for Continuous Media Applications", 6th International Workshop on Network and Operating Systems Support for Digital Audio and Video (NOSSDAV), April 1996.

[8] R. Sinha and C. Papadopoulos, "An Adaptive Multiple Retransmission Technique for Continuous Media Streams," Proc. ACM NOSSDAV, Cork, Ireland, Jun. 2004.

[9] H. Hagino, Y. Miyazaki, Y. Onoe, Y. Atsumi, H. Komaki, M. Taniguchi, N. Yamanouchi, "A Playout Time Oriented Retransmission Scheme for Multimedia Streaming Systems", HSNMC 2003, Estoril, Portugal, 23-25 July 2003., pp406-415

[10] H. Schulzrinne, S. Casner, R. Frederick, V. Jacobson, "RTP: A Transport Protocol for Real-Time Applications", Internet Engineering Task Force, RFC 1889, January 1996

[11] A. Miyazaki, H. Fukushima, K. Hata, T. Wiebke, R. Hakenberg, C. Burmeister, N. Taka-tori, S. Okumura, T. Ohno, " RTP Payload Formats to Enable Multiple Selective Re-transmissions", draft-ietf-avt-rtp-selret-05.txt, IETF, 2002

[12] J. Ott, S. Wenger, N. Sato, C. Burmeister, J. Rey, "Extended RTP Profile for RTCP-based Feedback
(RTP/AVPF)", draft-ietf-avt-rtcp-feedback-05.txt, IETF, 2003

[13] J. Rey, D. Leon, A. Miyazaki, V. Varsa, R. Hakenberg, "RTP Retransmission Payload Format", draft-ietf-avt-rtpretransmission-07.txt, IETF, 2003

[14] M. Feamster, H. Balakrishnan, "Packet Loss Recovery for Streaming Video", 12th International Packet Video Workshop, Pittsburgh, PA, April 2002.

[15] Árpád Huszák, Sándor Imre: "Source Controlled and Delay Sensitive Selective Retransmission Scheme for Multimedia Streaming", IEEE Symposium on Computers and Communications, ISCC 2007, ISBN 1-4244-1521-7, pp. 191-196., Aveiro, Portugal, 1-4 July, 2007

[16] Cormac J. Sreenan, Jyh-Cheng Chen, Prathima Agrawal, B. Narendran, "Delay reduction techniques for playout buffering", IEEE Transactions on Multimedia, pp88-100, June 2000 .

[17] J.-P. Wagner, P. Frossard, "Playback Delay Optimization in Scalable Video Streaming”, IEEE International Conference on Multimedia and Expo, ICME 2005, Amsterdam, The Netherlands, July 2005.

[18] V. Weerackody, G. Schuller,H.-L. Lou, "Streaming of multimedia with reduced start-up delay", IEEE International Conference on Communications, Helsinki, Finland, June 11-14, 2001

[19] P. Brady, "Effects of Transmission Delay on Conversational Behavior on Echo-free Telephone Circuits," Bell System Technical Journal, Vol. 50, No. 1, pp.115-134, January 1971.

[20] Ns-2 - Network Simulator, http:///www.isi.edu/nsnam/ns/index.html 